\title{
Morphological Characteristics of Psoriatic Lesions Affect the Accuracy and Reliability of Severity Assessments: Proposal for New Working Criteria for the Psoriasis Area and Severity Index
}

\author{
Chong Won Choi, Bo Ri Kim, Seungkul Yang, Sang Woong Youn \\ Department of Dermatology, Seoul National University College of Medicine, Seoul National University Bundang Hospital, Seongnam, \\ Korea
}

\section{Dear Editor:}

The recent introduction of biologics has increased the need for objective and reliable methods for assessment of the severity of psoriasis, to verify and compare the effectiveness of new agents ${ }^{1}$. The psoriasis area and severity index (PASI) is the most widely used severity assessment method in psoriasis ${ }^{1,2}$. As the severity components of the PASI (involved area, erythema, thickness, and scaling) are subjectively assessed by clinicians, the scoring of the PASI may reflect a physician's particular experience ${ }^{1,2}$. Thus, the PASI has some limitations in accuracy and reliability $^{3,4}$. In a previous study performed during a nationwide PASI educational workshop, 96 residents and board-certified dermatologists were participated and and 72 participants completed all the tests. The accuracy analyzed by counting the number of correct assessments of 10 images after the workshop was $5.54 \pm 2.17,5.54 \pm 2.17$, $5.38 \pm 2.15$, and $6.28 \pm 2.78$ in area, erythema, thickness, and scale, respectively. Despite presentation of educational lectures and reference photographs, we found that improvement in the accuracy and reliability of workshop participants for the PASI was not satisfactory ${ }^{5}$. In this study, we investigated the influence of morphological characteristics of psoriatic lesions on the accuracy and reliability of severity assessments, and suggest new working criteria for more objective and reliable PASI scoring. This study was approved by the Institutional Review Board of our hospital (IRB no. X-1608/360-902).

This is a post-hoc analysis of a previous study performed during the Korean Nationwide PASI Educational Workshop. To analyze the influence of morphological characteristics of psoriatic lesions on the accuracy and reliability of severity assessments, we examined the results of the previous study ${ }^{5}$. The test for severity assessment performed during the study consisted of five images of representative psoriatic lesions with different morphological characteristics (Table 1). The participants were asked to score the severity components (erythema, thickness, and scaling of psoriatic lesions, and the percentage of skin affected) of five images that were shown twice in random sequence. To rule out bias caused by lack of knowledge of the severity assessment methods, we used the results of the last test performed after the PASI educational lecture and the associated reference photograph as criteria for severity assessment. For the analysis of accuracy, the score assigned by the study participant was compared with that of a PASI expert who participated in the development of the educational lecture and test. In the assessment of involved

Received December 5, 2017, Revised February 13, 2018, Accepted for publication February 15, 2018

Corresponding author: Sang Woong Youn, Department of Dermatology, Seoul National University College of Medicine, Seoul National University Bundang Hospital, 82 Gumi-ro 173beon-gil, Bundang-gu, Seongnam 13620, Korea. Tel: 82-31-787-7319, Fax: 82-31-787-4058, E-mail: swyoun@snu.ac.kr ORCID: https:/orcid.org/0000-0002-5602-3530

This is an Open Access article distributed under the terms of the Creative Commons Attribution Non-Commercial License (http://creativecommons.org/ licenses/by-nc/4.0) which permits unrestricted non-commercial use, distribution, and reproduction in any medium, provided the original work is properly cited.

Copyright (C) The Korean Dermatological Association and The Korean Society for Investigative Dermatology 
Brief Report

Table 1. Characteristics of images used in the test

\begin{tabular}{ccccc}
\hline Image number & Type of psoriasis & Distribution of lesions & Erythema & Scale and thickness \\
\hline Image 1 & Small plaque & Even & Uniform & Uniform in scale \\
Image 5 & Large plaque & Even & Uniform & Uniform in thickness \\
Image 2 & Large plaque & Uneven & Uneven & Variable in thickness \\
Image 4 & Large plaque & Uneven & (two degrees of severity) & Uneven \\
Image 3 & Small plaque & Uneven & (some lesions were covered by scale) & Uneven in scale \\
& & & (two degrees of severity) & Variable in thickness \\
\hline
\end{tabular}
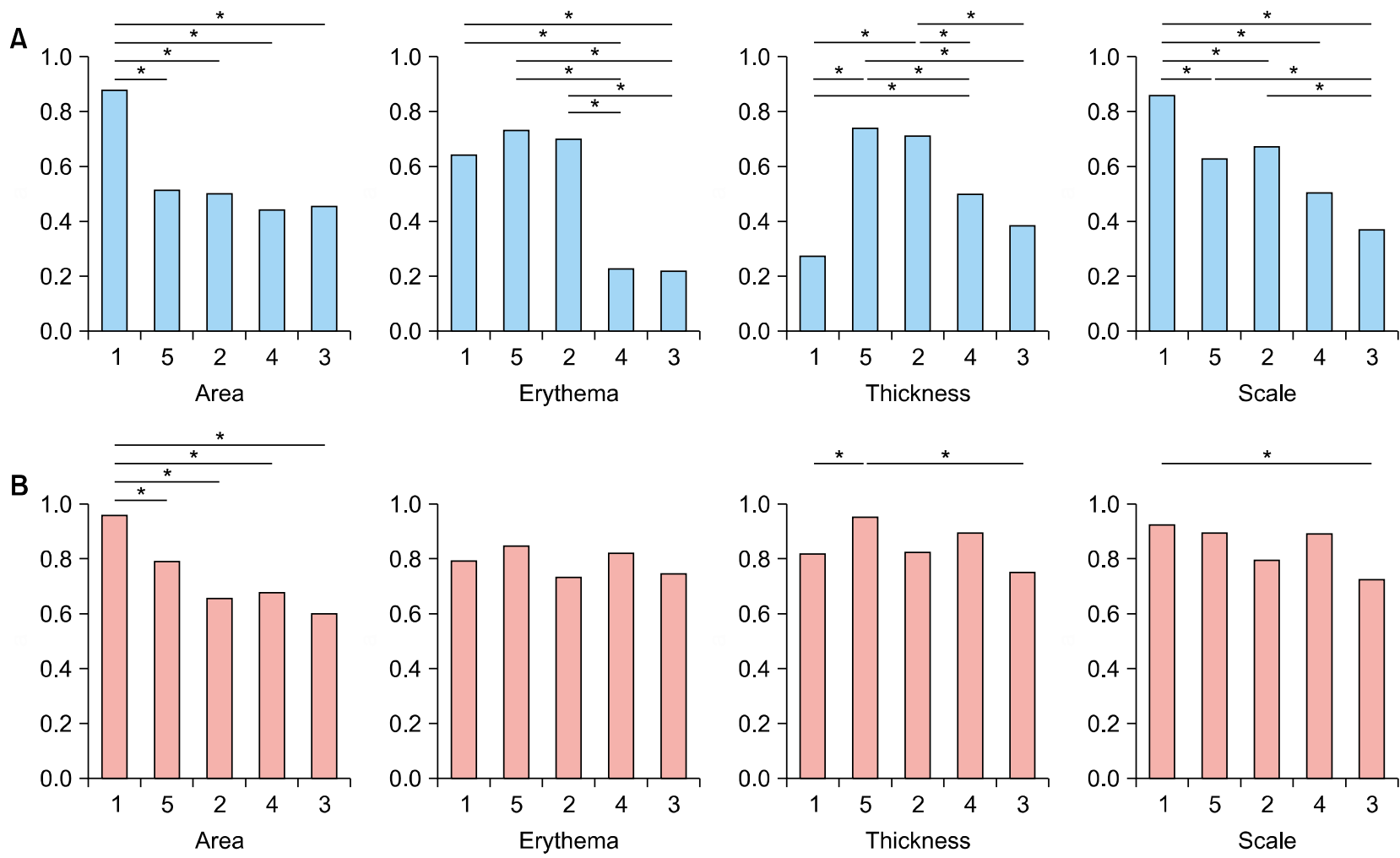

Fig. 1. Accuracy and reliability of severity assessment were influenced by the characteristics of psoriasis lesions. The accuracy for image 1 and image 5 was higher compared to those for images of non-uniform lesions such as image 4 and image 3 (A). The reliability for image 3 was lower compared to that for images of uniform lesions such as image 1 or image 5 in the assessment of involved area, thickness, and scale (B). Data were analyzed using the generalized estimating equation model to correct for repeated assessment of each image $\left({ }^{*} p<0.05\right)$.

area (percentage of skin affected by psoriatic lesions), we regarded the participant's response as correct when the difference between participant and expert scores was 5 or less. For other severity assessments (grades of erythema, thickness, and scaling), the assessment was regarded as correct when the score was the same as that of the PASI expert. For the analysis of reliability, two scores of the same image assessed by each participant were compared. In the assessment of involved area (percentage of skin affected by psoriatic lesions), we regarded the participant's assessments as identical when the difference between the scores for the same image was 5 or less. For other severity assessments (grades of erythema, thickness, and scaling), the assessment was regarded as identical when the scores for the same image were the same. To analyze accuracy and reliability, the proportion of correct assessments and the proportion of identical assessments were calculated and analyzed using the generalized estimating equation model to correct for repeated assessments of each image. IBM SPSS Statistics (IBM, Armonk, NY, USA) was used to 
perform statistical analysis and a $p$-value $<0.05$ was considered significant.

The results of the present study suggest that the morphological characteristics of psoriatic lesions have a significant influence on the accuracy and reliability of severity assessments. The accuracy and reliability for images with uniform lesions was higher compared with those for non-uniform lesions (Fig. 1). The results for image 1 and image 5 showed higher accuracy compared to those for images of non-uniform lesions such as image 4 and image 3 (Fig. 1A). In addition, the reliability for image 3 , which showed variable and non-uniform psoriatic lesions, was lower compared to that for images of uniform lesions such as image 1 or image 5 in the assessment of involved area, thickness, and scale (Fig. 1B). These results confirm that the uniformity of psoriatic lesions can affect the accuracy and reliability of severity assessment, and that the participant could have been confused by having to evaluate psoriatic lesions with variable degrees of severity in the same image. In addition, we found that the assessment of erythema, scaling, and thickness can interact with each other. In image 1, the accuracy of thickness assessment was low, where the scales covered the psoriatic lesions, whereas in image 5, the accuracy of thickness assessment was high, where the thickness of lesions was clearly seen due to absence of scale. We speculated that this was because scale covered the psoriatic lesions and caused confusion in scoring the degree of erythema and thickness.

In actual clinical practice, the psoriatic patient has multiple lesions with variable degrees of severity. However, working criteria used for scoring multiple lesions with variable degrees of severity are not well-established. Thus, many clinicians have chosen a representative psoriatic lesion among multiple lesions and evaluated the representative lesion to calculate the PASI score. However, the representative psoriatic lesion selected by each clinician may not be the same, and this may cause decreased accuracy and reliability of the PASI assessment. The decrease in accuracy and reliability is expected to be more profound in those who have little experience with PASI assessment. To overcome disagreement in choosing representative lesions, we suggest new working criteria for the severity assessment of multiple lesions with variable degrees of severity, based on the average severity of multiple lesions. However, this method will increase the time required for PASI assessment. In addition, we found that the evaluation of erythema and thickness was confused by the presence of scale. Therefore, to exclude the influence of scale for accurate severity assessments when scoring erythema and thickness, we suggest that the clinician needs to exclude the effect of scale in the assessment of erythema and thickness: scoring erythema and thickness of lesion without scale may be an alternative method. These working criteria can increase the accuracy and reliability of PASI assessments especially in those who have little experience with PASI assessment.

\section{ACKNOWLEDGMENT}

The authors thank Janssen Korea for organizing the nation-wide PASI educational workshops. The authors thank the Division of Statistics in the Medical Research Collaborating Center at Seoul National University Bundang Hospital for statistical analyses and also thank JY Lee and BR Yang in the Medical Research Collaborating Center at Seoul National University Hospital for advice on the development of the test.

\section{CONFLICT OF INTEREST}

The authors have nothing to disclose.

\section{ORCID}

Chong Won Choi, https://orcid.org/0000-0001-9994-8819

Bo Ri Kim, https://orcid.org/0000-0002-2223-1606

Seungkul Yang, https://orcid.org/0000-0003-4133-6638

Sang Woong Youn, https://orcid.org/0000-0002-5602-3530

\section{REFERENCES}

1. Choi CW, Kim BR, Seo E, Youn SW. The objective Psoriasis Area and Severity Index: a randomized controlled pilot study comparing the effectiveness of ciclosporin and methotrexate. Br J Dermatol 2017; 177:1740-1741.

2. Singh S, Pandey SS. Evaluation of efficacy of therapy for psoriasis : methodological issues. Indian J Dermatol Venereol Leprol 1997;63:211.

3. Armstrong AW, Parsi K, Schupp CW, Mease PJ, Duffin KC. Standardizing training for psoriasis measures: effectiveness of an online training video on Psoriasis Area and Severity Index assessment by physician and patient raters. JAMA Dermatol 2013;149:577-582.

4. Faria JR, Aarão AR, Jimenez LM, Silva $\mathrm{OH}$, Avelleira JC. Inter-rater concordance study of the PASI (Psoriasis Area and Severity Index). An Bras Dermatol 2010;85:625-629.

5. Choi CW, Kim BR, Park JS, Youn SW. Both educational lectures and reference photographs are necessary to improve the accuracy and reliability of Psoriasis Area and Severity Index (PASI) assessment: results from Korean nation-wide PASI educational workshop. Ann Dermatol 2018;30:284-289. 